\title{
Direct visualization of irreducible ferrielectricity in crystals
}

\author{
Kai $\mathrm{Du}^{1,5}$, Lei Guo ${ }^{2,5}$, Jin Peng ${ }^{2,5}$, Xing Chen ${ }^{1}$, Zheng-Nan Zhou' ${ }^{1}$, Yang Zhang $\mathbb{1}^{2}$, Ting Zheng ${ }^{2}$, Yan-Ping Liang ${ }^{2}$, Jun-Peng Lu $\mathbb{C}^{2}$,
} Zhen-Hua Ni ${ }^{2}$, Shan-Shan Wang ${ }^{2}$, Gustaaf Van Tendeloo ${ }^{3,4}$, Ze Zhang ${ }^{1}$, Shuai Dong $\mathbb{D}^{2 凶}$ and He Tian $\mathbb{D}^{1 凶}$

In solids, charge polarity can one-to-one correspond to spin polarity phenomenologically, e.g., ferroelectricity/ferromagnetism, antiferroelectricity/antiferromagnetism, and even dipole-vortex/magnetic-vortex, but ferrielectricity/ferrimagnetism kept telling a disparate story in microscopic level. Since the definition of a charge dipole involves more than one ion, there may be multiple choices for a dipole unit, which makes most ferrielectric orders equivalent to ferroelectric ones, i.e., this ferrielectricity is not necessary to be a real independent branch of polarity. In this work, by using the spherical aberration-corrected scanning transmission electron microscope, we visualize a nontrivial ferrielectric structural evolution in $\mathrm{BaFe}_{2} \mathrm{Se}_{3}$, in which the development of two polar sub-lattices is out-of-sync, for which we term it as irreducible ferrielectricity. Such irreducible ferrielectricity leads to a non-monotonic behavior for the temperature-dependent polarization, and even a compensation point in the ordered state. Our finding unambiguously distinguishes ferrielectrics from ferroelectrics in solids.

npj Quantum Materials (2020)5:49; https://doi.org/10.1038/s41535-020-00252-y

\section{INTRODUCTION}

Ferrielectricity, the equivalent of ferrimagnetism, can be termed as antiferroelectric order but with a switchable polarization, as sketched in Fig. 1a. The first proposed ferrielectric system was a composition of two antiferroelectric compounds $\mathrm{NaVO}_{3}$ and $\mathrm{NaNbO}_{3}{ }^{1}$, but subsequent experiments did not support this claim $^{2}$. Despite a long history, the existence of ferrielectricity in solid crystals remains rare, except in liquid crystals ${ }^{3}$. Recently, some solids were claimed to be ferrielectric. For example, in the layered-structure Culn $\mathrm{P}_{2} \mathrm{~S}_{6}$, the positions of $\mathrm{Cu}$ and In are always opposite within each unit cell (u.c.), which can be recognized as two uncompensated polar sub-lattices with antiparallel alignment ${ }^{4,5}$. In geometric ferroelectrics, such as $\mathrm{Ca}_{3} \mathrm{Ti}_{2} \mathrm{O}_{7}$ and some perovskite superlattices, the displacements of ions are opposite between layers, which can also been recognized as two uncompensated dipole moments ${ }^{6,7}$. Despite the macroscopic and phenomenological analogy as shown in Fig. 1a, it should be noted that there is a key difference between the ferrimagnetic and ferrielectric systems in the microscopic level. Different from the spin moment which can be defined on a single ion, the definition of a charge dipole involves more than one ion and thus may have multiple choices in ionic crystals. By choosing different ions as a dipole unit, multiple dipole values for a sub-lattice can be obtained $^{8}$. In this sense, the $\mathrm{Cu}$-In pair in $\mathrm{CulnP}_{2} \mathrm{~S}_{6}$ or bilayer in $\mathrm{Ca}_{3} \mathrm{Ti}_{2} \mathrm{O}_{7}$ can be treated as a dipole unit, which are indeed entangled simultaneously ${ }^{4,6}$. Therefore, these systems are indistinguishable from ferroelectrics, i.e., 'reducible' ferrielectrics, as qualitatively sketched in Fig. $1 \mathrm{a}$. Indeed, $\mathrm{CuInP}_{2} \mathrm{~S}_{6}$ only shows one paraelectric-ferroelectric transition temperature, and in most cases those geometric ferroelectrics are coined as 'ferroelectrics' rather than 'ferrielectrics's,9. Similar situations also exist for other ferrielectrics like $\mathrm{Pb}_{2} \mathrm{MnWO}_{6}{ }^{10}$. Conceptually different to these 'reducible' ferrielectrics, in this article, we provide proof of an 'irreducible' ferrielectricity (see Fig. 1a for its conceptual definition of 'irreducible' ferrielectricity) in $\mathrm{BaFe}_{2} \mathrm{Se}_{3}$. We will show unique ferri-characteristics clearly distinguishable from ferroelectricity.

\section{RESULTS}

$\mathrm{BaFe}_{2} \mathrm{Se}_{3}$ : ferrielectric material at room temperature

$\mathrm{BaFe}_{2} \mathrm{Se}_{3}$ belongs to the iron-based superconductor family ${ }^{11-13}$ but predicted to be multiferroic under ambient conditions ${ }^{14}$. As shown in Fig. 1b, a BaFe $\mathrm{Se}_{3}$ u.c. contains two iron ladders (labeled as $A$ and $B$ ). Long-range block-type antiferromagnetism (blockAFM) appears below the Néel temperature $T_{N} \sim 240-256 \mathrm{~K}^{15-19}$. The structural tetramerization due to the block-AFM leads to charge dipoles along the $a$-axis and the alignment of dipoles is almost antiparallel but with a tiny canting angle $\left(\sim 5.78^{\circ}\right.$ at room temperature) between ladders $A$ and $B$ (schematic displacements are shown in Fig. 1c $)^{14}$. It should be noted that all other members of the 123-series iron selenides (e.g., $\mathrm{BaFe}_{2} \mathrm{~S}_{3}$ ) also own a similar (quasi-) one-dimensional (1D) ladder structure, but only $\mathrm{BaFe}_{2} \mathrm{Se}_{3}$ has the canting ladder characteristic. According to theory ${ }^{14}, a$ residual polarization along the $c$-axis $\left(P_{c}\right)$ is expected in $\mathrm{BaFe}_{2} \mathrm{Se}_{3}$, as a characteristic of a 'reducible' ferrielectric material.

The tilting of the iron ladders gradually disappears with increasing temperature up to $\sim 600 \mathrm{~K}$, leading to a high symmetric $B b m m$ phase, according to X-ray diffraction ${ }^{16}$. Our in-situ selected area electron diffraction (SAED) results give distinctive patterns (Fig. 1c vs. Fig. 1d), confirming the disappearance of the ladder canting at high temperature. Differential Scanning Calorimetry (DSC) measurements (Fig. 1e) confirm the phase transition occurring at $\sim 610 \mathrm{~K}$ as a second-order one. Besides, there is a first-order phase transition at $\sim 420 \mathrm{~K}$, which was also evidenced in ref. ${ }^{16}$. but its real origin remains a puzzle. In addition, the resistivity behavior also supports this first-order transition (Supplementary Fig. 1). Since our neutron powder diffraction (NPD) data confirm that only one block-AFM transition appears at

\footnotetext{
${ }^{1}$ Center of Electron Microscopy, State Key Laboratory of Silicon Materials, and School of Materials Science and Engineering, Zhejiang University, 310027 Hangzhou, China. ${ }^{2}$ School of Physics, Southeast University, 211189 Nanjing, China. ${ }^{3}$ Electron Microscopy for Materials Science (EMAT), University of Antwerp, Groenenborgerlaan 171, B-2020 Antwerp, Belgium. ${ }^{4}$ Nanostructure Research Centre (NRC), Wuhan University of Technology, 430070 Wuhan, China. ${ }^{5}$ These authors contributed equally: Kai Du, Lei Guo, Jin Peng. 凶email: sdong@seu.edu.cn; hetian@zju.edu.cn
} 
a

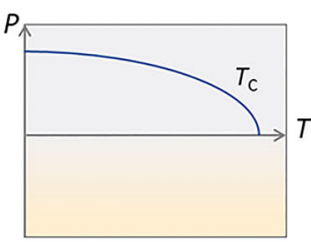

FE

b

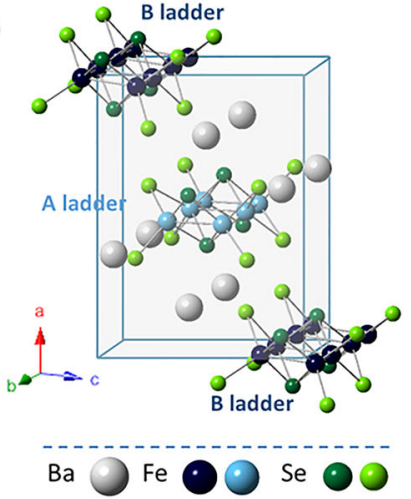

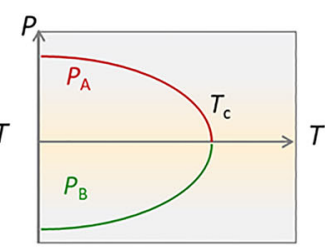

AFE

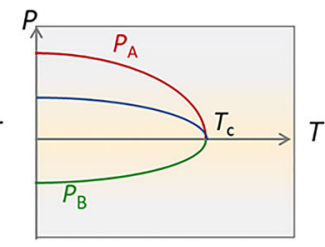

$r$-FiE/FE

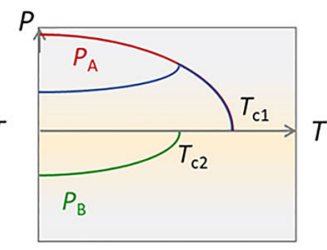

ir-FiE

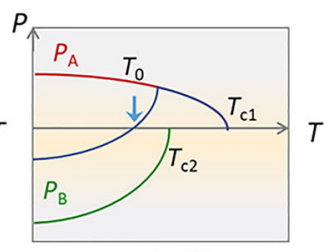

ir-FiE c

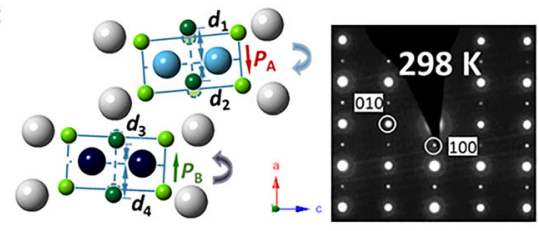

d

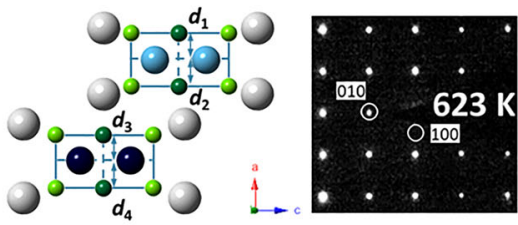

e

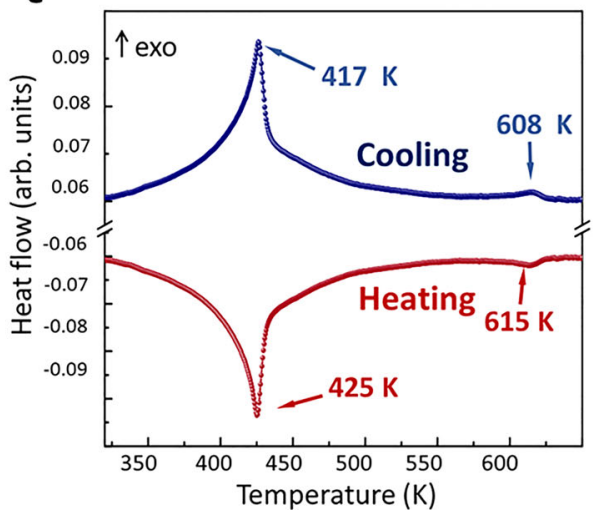

Fig. 1 Concept of ferrielectricity and candidate material $\mathrm{BaFe}_{2} \mathrm{Se}_{3}$. a Polarization $(P)$ vs. temperature $(T)$ of ferroelectricity (FE), antiferroelectricity (AFE), reducible-ferrielectricity ( $r$-FiE) and irreducible-ferrielectricity (ir-FiE), respectively. In $r$-FiE, two polar sub-lattices (A and B) develop synchronously, with one unique critical temperature. This type of ferrielectricity is macroscopically equivalent to ferroelectricity. In ir-FiE, the evolution of sub-lattices $\mathrm{A}$ and $\mathrm{B}$ is out-of-sync, with two critical temperatures (a higher $T_{\mathrm{C} 1}$ and a lower $T_{\mathrm{C} 2}$ ). A compensation point $\left(T_{0}\right)$ may appear in some ir-FiE systems. Note that any information on the order of the phase transition is not represented in these qualitative cartoons. b Crystal structure of $\mathrm{BaFe}_{2} \mathrm{Se}_{3}$. Each unit is composed of two iron-ladders. c Room temperature structure with a tiny tilting angle between the ladders A and B. d High temperature structure without tilting. The in-situ selected area electron diffraction patterns are shown. The lattice periodicity along the [100] direction changes from $11.90 \AA$ to $5.96 \AA$ as the temperature rises from $298 \mathrm{~K}$ to $623 \mathrm{~K}$. e DSC curves indicate two transitions at $\sim 610 \mathrm{~K}$ and $\sim 420 \mathrm{~K}$. The $\sim 610 \mathrm{~K}$ transition is a second-order one with a step-like behavior, while the $\sim 420 \mathrm{~K}$ transition is a first-order one with a peak.

$250 \mathrm{~K}$, obviously, the transition at $\sim 420 \mathrm{~K}$ can be excluded as a magnetic-ordering behavior (Supplementary Fig. 2a).

Using a spherical aberration-corrected scanning transmission electron microscope (Cs-STEM), we were able to determine the subtle structure evolution of the 1D Fe chains, as well as the associated $\mathrm{Se}^{\prime} \mathrm{s}$. Then the structure of $\mathrm{BaFe}_{2} \mathrm{Se}_{3}$ can be directly measured atom column by atom column, which reveals an unexpected irreducible ferrielectricity going beyond the theoretical expectations. The STEM image of $\mathrm{BaFe}_{2} \mathrm{Se}_{3}$ along $b$-axis and $c$ axis was shown in Supplementary Fig. 3. The STEM results at room temperature are summarized in Fig. 2. Interestingly the structural tetramerization already exists at room temperature and, unexpectedly, the intensities for ladders $A$ and $B$ are not equivalent, creating a strong ladder and a weak ladder within a unit cell. The inequivalent features in strong ladders and weak ladders are embodied in magnitude of displacements, as shown schematically in Fig. $2 a$ and in the high-angle annular dark-field scanning transmission electron microscopy (HAADF-STEM) images along the $b$ (Fig. 2b, d) and $c$ axis (Fig. 2c, e). The line profiles along these two ladders are shown in Supplementary Fig. 4. A displacement vector-mapping algorithm was implemented on the crosssectional HAADF-STEM images to measure the local displacement of the atoms. Based on the statistics of about 300 data for each length, the Fe atoms displacement in the strong ladders are stronger than that in the weak ladders (see Supplementary Fig. 5 for an example). Considering that the Fe-block tetramerization could induce Se ions displacement along the $a$-axis ${ }^{14}$ (as clearly indicated in Fig. 2b, d), such inequivalence of Fe displacements will lead to a residual polarization along the $a$-axis $\left(P_{a}\right)$, which is larger than the expected $P_{c}$. The arrow is added to make it more visible. The uncovered zoom-in images (raw data) have been shown in Fig. 2(b, c). Therefore, $\mathrm{BaFe}_{2} \mathrm{Se}_{3}$ is a room temperature ferrielectric with polarization mainly along the $a$-axis, rather than the expected low temperature ferrielectric with polarization along the $c$-axis ${ }^{14}$.

To explore the origin of this unbalanced ladders, monochromated electron energy loss spectra (mono-EELS) were acquired to demonstrate the underlying charge modulation as shown in Fig. 2f. Using the monochromatror we reach an energy resolution of $0.3 \mathrm{eV}$, which is enough to detect subtle changes in the fine structure of the EELS excitation edges. The averaged Fe- $L_{3}$ edges in strong chains and in weak chains show significant differences in their ELNES. Comparing with Fe reference spectra ${ }^{20}$, the valence states of the strong and the weak chains in $\mathrm{BaFe}_{2} \mathrm{Se}_{3}$ are different, and the Fe- $L_{3}$ peak shift between them is approximately $0.4 \mathrm{eV}$ (shown as the distance between two dashed line in Fig. 2f). Note that the EELS have an ability to reflect a valance change, but the absolute valance identification is still challenging, since the absolute energy position always has several hundred meV uncertainty. Such charge disproportion is not unusual in correlated electron systems, especially in Fe-based oxides and fluorides, e.g., $\mathrm{Fe}_{3} \mathrm{O}_{4}$ and $\mathrm{LiFe}_{2} \mathrm{~F}_{6}{ }^{21,22}$, although it has not been reported in selenides before. Nominally, the valences of Fe can become $+(2+\delta)$ and $+(2-\delta)$ for the two sublattices, and a proper $\delta$ (around 0.15 according to our fitting results) can lead to the structural tetramerization following the idea of Peierls transition $^{14}$. It should be noted that the non-integer valences are possible in iron selenides, e.g., in $\mathrm{KFe}_{2} \mathrm{Se}_{3}$ and $\mathrm{KFe}_{2} \mathrm{Se}_{2}{ }^{23-25}$. Therefore, charge-ordering, i.e., difference of local electron density, can be the key ingredient for the unbalanced structural tetramerization and affiliated polarization, which needs deeper investigation in future. 
a

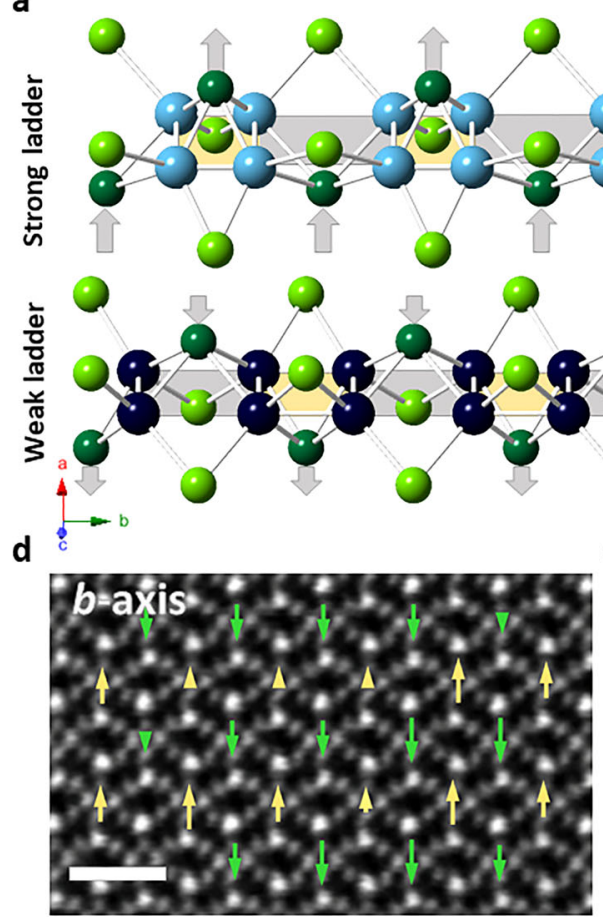

b

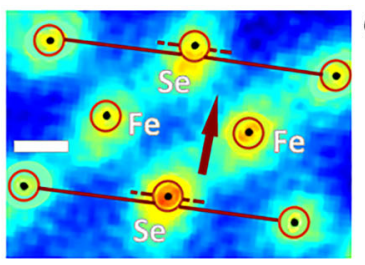

Strong ladder

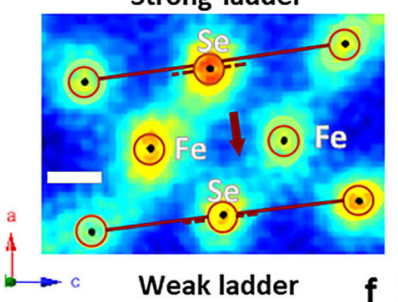

e
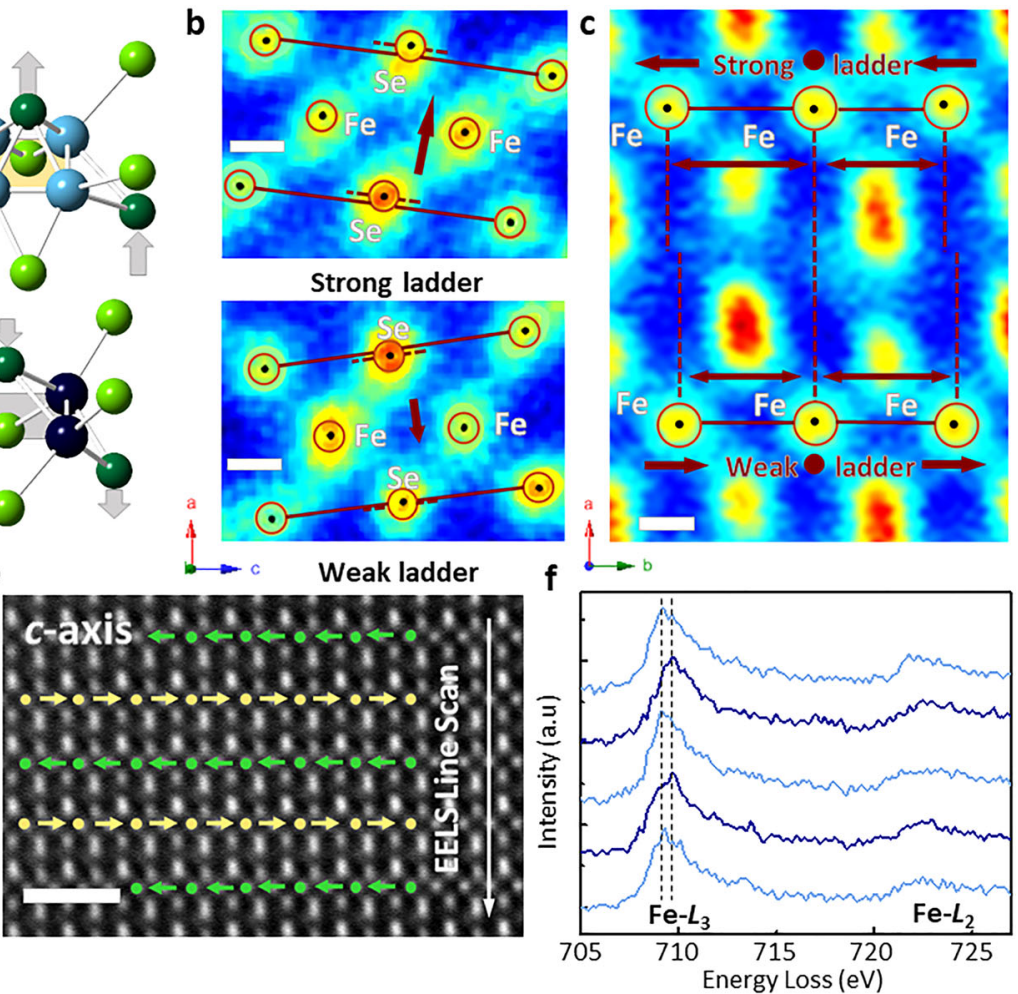

Fig. 2 Atomic displacements in the ladders. a Schematic displacements of Fe and Se ions indicated by arrows and amplified in magnitude. Light blue: strong ladder with larger polar bias of Se ions; Dark blue: weak ladder. $\mathbf{b}, \mathbf{c}$ Color-enhanced HAADF-STEM images of BaFe $\mathrm{Se}_{3}$ along $b$-axis and $c$-axis. The strong and weak ladders are distributed in an alternating order. Scale bar, $1 \AA$. d, e Superposition of the HAADF image and the polar map of Se atoms along $b$-axis and Fe atoms along $c$-axis. The yellow and green arrows there represent the atoms displacements in strong and weak ladders, respectively. Scale bar, $1 \mathrm{~nm}$. $\mathbf{f}$ The EELS line scan is across the white line in e. EELS measurements indicate the charge ordering pattern. The averaged Fe $L_{2,3}$ edge spectrum from neighboring Fe chains is presented. A Fe- $L_{3}$ peak shift of approximately $0.4 \mathrm{eV}$ between neighboring Fe chain is observed.

Variation of the $\mathrm{BaFe}_{2} \mathrm{Se}_{3}$ structure with various temperature

Then it is interesting to know its ferrielectric $T_{\mathrm{C}}$. To characterize the structural tetramerization, the difference $(\delta)$ of nearest-neighbor Fe-Fe bond length $(d)$ along the ladder direction is measured as a function of temperature (Fig. 3a). An in-situ heating experiment was performed using a DENSsolutions $\mathrm{SH} 30$ system to be able to measure over a wide temperature range. A lamella of $\mathrm{BaFe}_{2} \mathrm{Se}_{3}$ was transferred onto specialized chips using a probe-assistance method (Supplementary Fig. 6), then the sample was heated to the set temperature by resistance heating. The displacement vector-mapping algorithm was implemented on the STEM images, and each $\delta$ is obtained by averaging around 300 measurements. The difference of Fe-Fe bond length, as well as the tilting angle between the ladders at high temperature vanishes $\sim 600 \mathrm{~K}$, and the in-ladder tetramerization almost drops to zero, implying a high symmetric nonpolar phase (in agreement with the DSC curve shown in Fig. 1e and the X-ray data in ref. ${ }^{16}$ ). The discrepancy between strong and weak ladders disappears in this high symmetric phase, as also demonstrated by above SAED result (Fig. 1d). With decreasing temperature (e.g., at $473 \mathrm{~K}$ ), unexpectedly, tetramerization emerges in one sublattice of ladders but not in the other, which is a unique characteristic of irreducible ferrielectricity, leading to an emergence of $P_{\mathrm{a}}$ (Fig. 3b) [calculated by density functional theory (DFT) $]^{26}$. Meanwhile, the ladders become tilting (Fig. 3c compared with Fig. 3d). With further decreasing temperature (e.g., at $423 \mathrm{~K}$ ), tetramerization emerges in both ladders with different intensities and slopes, implying that the first-order transition occurring at $\sim 420 \mathrm{~K}$ corresponds to the starting of tetramerization of the weak ladders. At $\sim 373 \mathrm{~K}$, the intensities of tetramerization are close to identical between both ladders, resulting in an (almost) canceled $P_{a}$, i.e., the unique compensation point $\left(T_{0}\right)$ of irreducible ferrielectricity. Below $T_{0}$, the difference between the two ladders increases with decreasing temperature, leading to a reentrance of $P_{a}$. It is worth to emphasize that the direction of polarization (i.e., the roles of strong and weak ladders) may be probably reversed across $T_{0}$ according the tendency (see the last panel of Fig. 1a), although our variable temperature measurements can not continuously track the evolution of individual ladder over so large temperature range. If so, the strong ladder $A$ (weak ladder $B$ ) becomes the weak ladder $\mathrm{A}$ (strong ladder $\mathrm{B}$ ) when temperature crossing $T_{0}$. More color-enhanced HAADF-STEM images acquired at different temperatures along the c-axis are shown in Supplementary Fig. 7. Statistics of the deviation of Fe-Fe bond length in the strong and weak chain at different temperatures could be seen in Supplementary Table 1.

Since the ferrielectric polarization can not be directly measured using electrical methods (and even pizeoelectric force microscopy) in the current stage due to the high leakage of the samples (considering the very small band gap $\sim 0.13-0.178 \mathrm{eV}^{17,18}$ ), an optical second harmonic generation (SHG) experiment is employed to characterize the polarity of the materials (see "Methods" section for experimental details). Our SHG signal (Fig. 3b) demonstrates its polarity below $\sim 600 \mathrm{~K}$. Most importantly, the non-monotonic evolution of the SHG signal unambiguously matches the DFT calculated polarization, including the possible compensation point at $\sim 380-400 \mathrm{~K}$, which provides a very strong evidence to support our STEM data.

The irreducible ferrielectricity of $\mathrm{BaFe}_{2} \mathrm{Se}_{3}$ can be qualitatively described by Landau theory with a coupling between two ladders $\left(P_{\mathrm{A}}\right.$ and $\left.P_{\mathrm{B}}\right)$. The most simplified free energy formula can be 

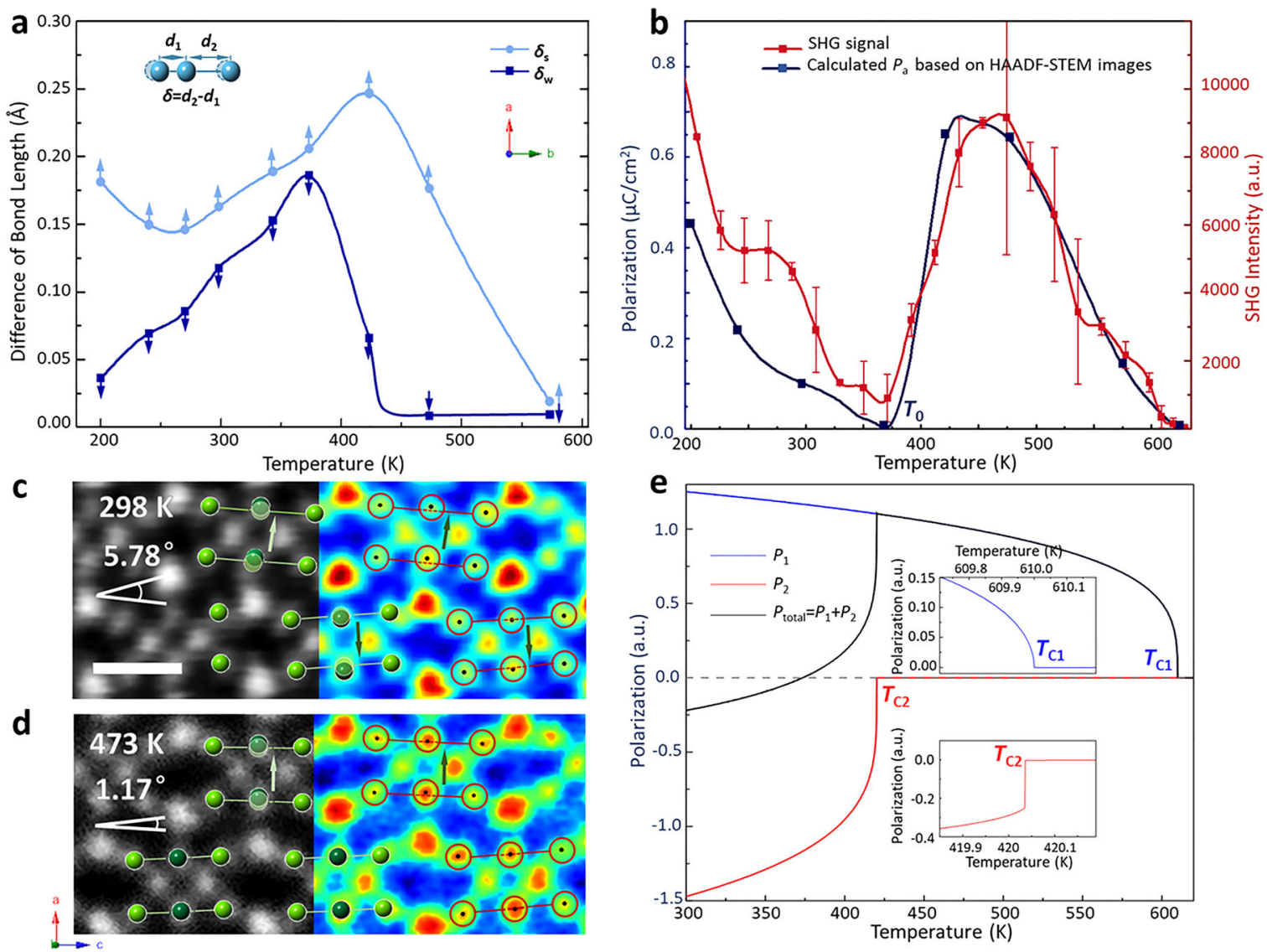

Fig. 3 Temperature-dependent evolution of $\mathrm{BaFe}_{2} \mathrm{Se}_{3}$. a The measured structural tetramerization. The light blue and dark blue lines correspond to the strong and weak ladders, respectively. The affiliated arrows denote the corresponding dipoles. b Left axis: the polarization along the $a$-axis (DFT calculated using the STEM structural data). Right axis: the SHG signal, which mainly reflects the evolution of $P_{\text {a }}$. The overall evolutions of the polarization and SHG signal qualitatively match. More SHG data and explanation can be found in Supplementary Figs. 8, Fig. 10. c, d Color-enhanced HAADF-STEM images acquired at $298 \mathrm{~K}$ and $473 \mathrm{~K}$ along the $b$-axis. The Se atoms displacement in the strong chain is larger than that in the weak chain at $298 \mathrm{~K}$; this is consistent with the difference of the Fe chain evolution magnitude at $298 \mathrm{~K}$. Also, the tilt angle between ladders varies from $5.78^{\circ}$ to $1.17^{\circ}$ at high temperature. Scale bar, $5 \AA$. e Simulated evolution of order parameters with a most simplified Landau-type free energy formula. Inserts: the magnified views near the phase transitions, which indicate a secondorder transition at $T_{\mathrm{C} 1}$ and a first-order transition at $T_{\mathrm{C} 2}$.

written as:

$$
\begin{aligned}
F= & a_{\mathrm{A}}\left(T-T_{\mathrm{A}}\right) P_{\mathrm{A}}^{2}+\beta_{\mathrm{A}} P_{\mathrm{A}}^{4}+\gamma_{\mathrm{A}} P_{\mathrm{A}}^{6} \\
& +a_{\mathrm{B}}\left(T-T_{\mathrm{B}}\right) P_{\mathrm{B}}^{2}+\beta_{\mathrm{B}} P_{\mathrm{B}}^{4}+\gamma_{\mathrm{B}} P_{\mathrm{B}}^{6}+a_{\mathrm{AB}} P_{\mathrm{A}} \cdot P_{\mathrm{B}},
\end{aligned}
$$

where the first to six items are the standard Laudau-GinzburgDevonshire type energy expression up to the sixth power for sublattices $A$ and $B$, while the last item is the antiferroelectric coupling between two sub-lattices. All coefficients except $\beta_{2}$ are positive and the small canting angle between $P_{\mathrm{A}}$ and $P_{\mathrm{B}}$ is neglected. Without fine tuning of the coefficients (see "Methods" section for details), the simulated evolution of the polarization (Fig. 3e) is qualitatively reproducing the non-monotonic experimental behavior, implying the correct main physics captured in the model. Consistent with the DSC data, the high temperature transition is a second-order one, while the low temperature transition is a firstorder one due to the negative $\beta_{2}$.

Manipulation of $\mathrm{BaFe}_{2} \mathrm{Se}_{3}$ by an external electric field As a polar material, electric field tuning of the polarization is a fundamental function. However a direct measurement of the electric hysteresis loop is technically challenging in the current stage due to serious leakage, but we have successfully developed an inspiring in-situ technique to manipulate the polar structure at an atomic scale by applying an electric field. An electrical bias was applied between a tungsten tip, which acts as a mobile electrode, and the lamella of $\mathrm{BaFe}_{2} \mathrm{Se}_{3}$, which is connected to ground (as schematically shown in Fig. 4a). To capture the changing of atoms positions, the real-time crystal structure with atomic-scale spatial resolution was characterized in STEM mode; the access to dynamic structural information provide a clear picture of the evolution under an external electric field. As shown in Fig. 4b, c and Supplementary Fig. 9, the tetramerization is significantly enhanced by applying an electric field along the $a$-axis. This implies a significant enhancement of polarization under the electric field. However, the reversal of $P_{\mathrm{a}}$ has not been achieved, implying a large coercive field.

Previous experiments reported the space group Pnma for $\mathrm{BaFe}_{2} \mathrm{Se}_{3}$ at room temperature ${ }^{15-17}$, which is nonpolar and does not allow the tetramerization. A recent work reported the space group Pmn2 1 (a subgroup of Pnma) at room temperature, allowing the tetramerization and polarization ${ }^{27}$. Another recent $\mathrm{X}$-ray diffraction work reported the space group $P m n 2_{1}$ at $300 \mathrm{~K}$ but $\mathrm{Pm}$ (a subgroup of $P m n 2_{1}$ allowing the inequivalent ladders) at $150 \mathrm{~K}^{28}$. In fact, the patterns of NPD (or X-ray) are very subtle among these space groups (see Supplementary Fig. 10 and Supplementary Fig. 11), which thus can not distinguish these structures precisely. Instead, our STEM technique is more suitable to monitor these subtle distortions of inner coordinates. According to our studies, below $T_{\mathrm{C} 1}$ the accurate space group should already 

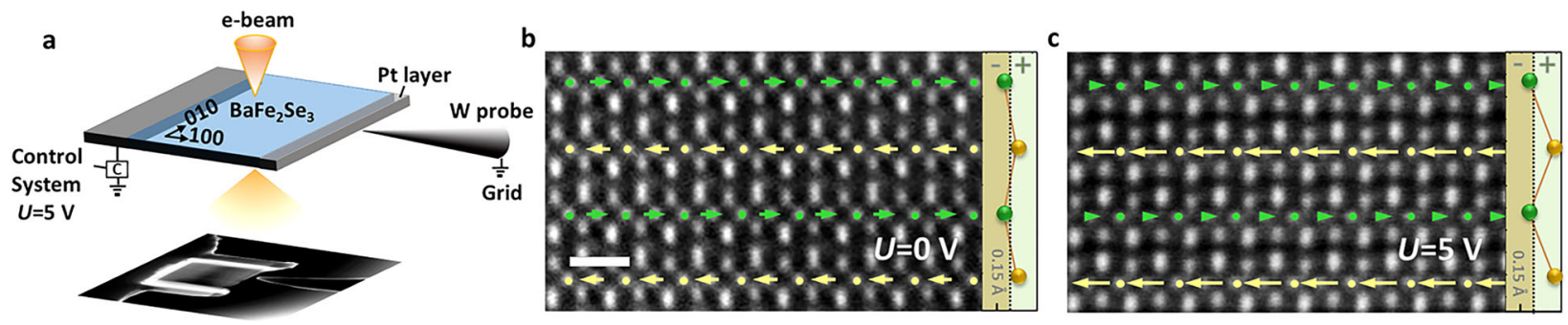

Fig. 4 Manipulation of the ferrielectric polarization in $\mathrm{BaFe}_{2} \mathrm{Se}_{3}$. a Schematic diagram of the electric field experiment. The in-situ biasing experiment was performed with an input voltage $V_{D C}=5 \mathrm{~V}$. To capture the structural changes, a real-time analysis is performed in STEM mode. b, c Comparison of the local dipoles with/without applied voltage. The arrows denote the displacements of $50 \%$ of the Fe ions, while the rest of the Fe ions are treated as reference points. The difference between the strong/weak ladders is further enhanced by the electric field. Arrows are added to make the displacements more visible. The uncovered zoom-in image (raw data) can be found in Fig. S9. Scale bar, $5 \AA$.

be $P m$, allowing the asymmetry between two ladders. The transition from $B b m m$ to $P m$ at $T_{\mathrm{C} 1}$ is a second-order one while the first-order transition at $T_{\mathrm{C} 2}$ does not change the symmetry. Other high-resolution technique, such as synchrotron X-ray diffraction, may be helpful to verify our STEM results in future.

\section{DISCUSSION}

Finally, it should be noted that the irreducible ferrielectrics is not limited to $\mathrm{BaFe}_{2} \mathrm{Se}_{3}$ but with broader interests. For example, as an important branch of multiferroics, $\mathrm{TbMn}_{2} \mathrm{O}_{5}$ and other 125-type manganites showed strange 'ferroelectric' behavior of polarization as a function of temperature or magnetic field ${ }^{29,30}$, including the compensation point of polarization. The real mechanism is that the ferroelectric contributions in $\mathrm{TbMn}_{2} \mathrm{O}_{5}$ are from three out-ofsync sources according to the SHG measurement ${ }^{30}$.

Even though, our current work on $\mathrm{BaFe}_{2} \mathrm{Se}_{3}$ is not a marginal extension of $\mathrm{TbMn}_{2} \mathrm{O}_{5}$. The polarity in $\mathrm{TbMn}_{2} \mathrm{O}_{5}$ is magnetismdriven, i.e., it is a so-called type-II multiferroic material, instead of a proper ferroelectric material. It is not rare for a magnetic system to have sequential magnetic phase transitions. In this sense, the nontrivial evolution of polarization in $\mathrm{TbMn}_{2} \mathrm{O}_{5}$ is just a secondary effect of magnetic evolution, which occurs at very low temperature $(<40 \mathrm{~K})$ and gives a very weak signal of polarization $\left(\sim<0.04 \mu \mathrm{C} / \mathrm{cm}^{2} \text { and } \sim<0.15 \mu \mathrm{C} / \mathrm{cm}^{2}\right)^{29,30}$. In our case, the ferrielectricity is not magnetism-driven but a primary polar property, which occurs above room-temperature ( 15 times of $\mathrm{TbMn}_{2} \mathrm{O}_{5}$ ) and with a much larger polarization (5-15 times of $\mathrm{TbMn}_{2} \mathrm{O}_{5}$ ). In addition, limited by its very weak polarization signal, the experimental measurements of $\mathrm{TbMn}_{2} \mathrm{O}_{5}$ can only rely on the pyroelectric method, which can lead to a net polarization but the microscopic facts of different contributions were mostly by suspecting or indirect derivation from SHG signals. Instead, our current work, powered by the advanced in-situ STEM techniques and thanks to the strong signal of $\mathrm{BaFe}_{2} \mathrm{Se}_{3}$, the microscopic evolution of two contributions can be visualized directly, leading to a more decisive conclusion. In fact, although the nontrivial polarization of $\mathrm{TbMn}_{2} \mathrm{O}_{5}$ has been known for decades, it is more likely to be recognized as a type-II multiferroics with strange ferroelectric behavior. Our work will lead to a re-look at the irreducible ferrielectricity, including that in $\mathrm{TbMn}_{2} \mathrm{O}_{5}$.

The irreducible ferrielectricity combines both characteristics of ferroelectricity and antiferroelectricity, making these systems having more degrees of freedom to be controlled. For example, by tuning the amplitudes of sub-lattice polarizations near the compensation point, the macroscopic polarization can be easily switched, without the reversal process of dipole moments as required in ferroelectric cases. Moreover, complex ferroelectric +antiferroelectric domain structures may be expected in ferrielectrics ${ }^{31}$, which deserve further studies.

In summary, employing spherical aberration-corrected STEM with sub-angstrom resolution, the structural evolution of $\mathrm{BaFe}_{2} \mathrm{Se}_{3}$ has been characterized in detail. Highly interesting phenomena, beyond previous experimental observations and theoretical predictions, have been detected and analyzed. First, $\mathrm{BaFe}_{2} \mathrm{Se}_{3}$ is a room temperature polar material. Second, combined with EELS analysis, the origin of its structural tetramerization is demonstrated to be driven by the local electron density, not the previously expected block-type antiferromagnetism. Third, most importantly, the evolution of the two ladders in $\mathrm{BaFe}_{2} \mathrm{Se}_{3}$ does not behave synchronously, leading to irreducible ferrielectricity. The compensation point, a unique fingerprint of irreducible ferrielectricity, is observed. The irreducible ferrielectricity reported here is conceptually different from previously reported reducible ferrielectricity which is actually equal to ferroelectricity. The irreducible ferrielectricity in $\mathrm{BaFe}_{2} \mathrm{Se}_{3}$ acts as the primary effect, leading to a stronger impact to the community to re-investigate this independent branch of polarity. More functionalities are promisingly expected in future based on irreducible ferrielectricity, e.g., the magnetic-field-tunable polarization as demonstrated in $\mathrm{TbMn}_{2} \mathrm{O}_{5}$.

\section{METHODS}

Material synthesis

High-quality $\mathrm{BaFe}_{2} \mathrm{Se}_{3}$ single crystals were grown by the self-flux technique starting from an intimate mixture of Ba pieces, Fe granules, and $\mathrm{Se}$ powders with an atomic ratio of 1:2:3. Then the starting materials were put in a carbon crucible and sealed in the quartz tube with partial pressure of argon. The quartz tube was first heated to $420^{\circ} \mathrm{C}$ at a rate of $1^{\circ} \mathrm{C} / \mathrm{min}$, held for $12 \mathrm{~h}$, and then annealed at $1150^{\circ} \mathrm{C}$ for another $24 \mathrm{~h}$. After that, the quartz tube was slowly cooled down to $750^{\circ} \mathrm{C}$ at a rate of $3{ }^{\circ} \mathrm{C} / \mathrm{h}$. Finally, the quartz tube was cooled down to room temperature naturally and the strip-like $\mathrm{BaFe}_{2} \mathrm{Se}_{3}$ single crystals with a typical size of $3.0 \times 1.0 \times 0.5 \mathrm{~mm}^{3}$ and shiny surfaces can be obtained.

\section{Macroscopic properties measurements}

Differential scanning calorimetry (DSC) experiments were performed with Maia DSC 200 F3. Measurements were performed on heating and cooling with a rate of $10 \mathrm{~K} \cdot \mathrm{min}^{-1}$. The sample is encapsulated in a standard Al crucible using argon stream as the protecting gas. XRD measurements were performed on Rigaku Smartlab3 with Cu $K_{a}$ radiation. In the SHG measurements, the incident laser with a wave length of $800 \mathrm{~nm}$ is perpendicular to the cleavage (100) plane and the reflected light at $400 \mathrm{~nm}$ is collected. The polarization of the incident laser is controlled by a half wavelength plate. Then the canting polarization along the $c$-axis can be monitored, which is in proportional to the main component of polarization along the $a$-axis. The SHG signal in Fig. $3 \mathrm{~b}$ was measured with the polarization of the incident laser (i.e., the electric field component $E$ ) in the bc cleavage surface (Fig. S10c) of the $\mathrm{BaFe}_{2} \mathrm{Se}_{3}$ crystal, while the angulardependent results with rotating $E$ can be found in Fig. S8. Magnetic measurements were carried out in a vibrating sample magnetometer (VSM) integrated in a Physical Property Measurement System (PPMS-9, Quantum Design) up to $600 \mathrm{~K}$. Neutron Powder Diffraction (NPD) data were collected on a High-intensity Powder Diffractometer Wombat at Australian Nuclear Science and Technology Organization (ANSTO) with 
$\lambda=2.41 \AA$, between $10 \mathrm{~K}$ and $500 \mathrm{~K}$. Resistivity was measured using Keithley $4200 A-5 C S$.

\section{Conventional and scanning transmission electron microscopy}

Samples was cut into lamellas by Focused Ion Beam (FEI Quanta 3D FEG) for the observation of electron microscope. We use spherical aberration correction electron microscopy (FEI Titan G2 80-200 ChemiSTEM, $30 \mathrm{mrad}$ convergence angle, $0.8 \AA$ spatial resolution) to acquire atomic resolution HAADF-STEM images of $\mathrm{BaFe}_{2} \mathrm{Se}_{3}$ 's cross section from different directions and the image noise was corrected using Digital Micrograph. All STEM images in this work are filtered in Fourier space using a grid mask to select for the lattice frequencies and by low and high pass annular filters to remove the zero frequency and high frequency noise above the information transfer limit. Electron Energy Loss Spectroscopy (EELS) test was also performed on $\mathrm{BaFe}_{2} \mathrm{Se}_{3}$ to verify whether there are changes in the valence state of iron. SAED patterns (selected area electron diffraction) obtained on FEI Tecnai G2 F20 S-TWIN are used to verify the analysis on the local evolution in the statistical sense. Some additional details should be mentioned: (1) As to exclude the influence of microscope artifacts, some steps have been taken. To minimize the influence of sample drift, most of the microscopy data for quantitative analysis are acquired under the condition of drift below 1 A $\mathrm{min}^{-1}$. (2) The STEM image were acquired from the mutually perpendicular directions, and the analysis results of bond length show no obvious difference between them. Thus, the possibility of STEM scanning direction as the main origination of the observed phenomenon can be excluded. (3) High frequency vibration of imaging would be another potential influence factor. Therefore, a technique of ultra-fast acquirement was employed. Tens of images were quickly acquired in the same local region, and most HAADF images shown in this article are overlaid based on such image series, the effect of the specimen drift and beam vibration were significantly reduced and the signal-to-noise ratio of the HAADF images was improved, simultaneously. (4) Aiming at avoiding oxidation, experiments were performed as soon as $\mathrm{BaFe}_{2} \mathrm{Se}_{3}$ was taken out of glove box which offer protecting gas. (5) In consideration of that potential slight damage caused by ion beam in FIB, we minished parameters including voltage and electric current of ion beam down to $2 \mathrm{kV} / 27 \mathrm{pA}$ to minimize the negative and unnecessary surface damage. For $\mathrm{BaFe}_{2} \mathrm{Se}_{3}$, the antiferromagnetic ordering temperature is $250 \mathrm{~K}$ (Supplementary Fig. 2), while most of STEM data are measured far above this temperature. Thus, the magnetic fields (from Cs-STEM) effect to polar distortion is negligible in the high temperature paramagnetic region.

\section{In-situ study}

The in-situ heating experiment was done on DENS solutions SH30 system in order to carry out the experiment in a wide temperature range. The NanoChip we used could control the temperature environment locally on the device via the 4-point-probe. Its highest temperature accuracy and stability is $0.001{ }^{\circ} \mathrm{C}$. The experimental data at low temperature was obtained by a demo low-temperature sample holder made by DENS. The in-situ biasing was done on Hysitron PI-95 TEM Picolndenter, the input voltage $V_{D C}=5 \mathrm{~V}$ was applied between the sharp conductive tip and the sample using a function generator. To capture the changing of atoms positions, the realtime crystal structure is characterized in STEM. The atomic-scale spatial resolution of STEM and the access to structural information provide a clear picture of the evolution under external electric field.

\section{Specimen transfer onto biasing chips}

To measure the performance of $\mathrm{BaFe}_{2} \mathrm{Se}_{3}$, as shown in Supplementary Fig. 6. After conventional FIB process of welding the lamella onto needle and transfer it near the surface of chips, additional confined Pt pad was deposited to contact the lamella onto the chip surface. The lamella thickness was to about $2 \mu \mathrm{m}$. Low current down to $10 \mathrm{pA}$ was used to polish the surface of chips after detaching the needle to reduce the amount of redeposited material resulting from the previous contacting process.

\section{DFT calculation}

The DFT calculation was performed based on the projector augmentedwave (PAW) potentials and Perdew-Burke-Ernzerhof exchange function as implemented in Vienna ab initio simulation package (VASP) ${ }^{32-34}$. The plane-wave energy was $500 \mathrm{eV}$. The experimental structures at different temperatures were used and the Cx-type antiferromagnetism is adopted for simplify (since here the polarization is not driven by magnetism). Brillouin zone integration was obtained using a $6 \times 3 \times 4 k$-point mesh. The standard Berry phase method is adopted to estimate the ferroelectric polarization ${ }^{35}$, while the intuitive point-charge-model provides similar results.

\section{Laudau-Ginzburg-Devonshire model}

To fit the experimental phase transitions, $T_{\mathrm{A}}=610 \mathrm{~K}$ and $T_{\mathrm{B}}=420 \mathrm{~K}$, are used. Noting $T_{\mathrm{C} 1}=T_{\mathrm{A}}$ for the second order phase transition, and $T_{\mathrm{C} 2}$ is close but slightly higher than $T_{\mathrm{B}}$ for the first order transition. To simulate Fig. $3 e, a_{1}=1$ as the unit, and $\beta_{1}=5, a_{2}=4.5, \beta_{2}=-5, \gamma_{1}=\gamma_{2}=40$. The negative $\beta_{2}$ is essential for the first-order transition around $T_{C 2}$ and the differences between $a_{1} / a_{2}, \beta_{1} / \beta_{2}$ originate from the charge disproportion. $a_{12}$ can be a small quantity, e.g., 0.001 .

\section{Determining the position of atoms}

Polarization mapping here was performed by calculating ion displacements in HAADF-STEM images. On account of the fact that the bright area of every atom in HAADF image is actually too large for us to determine where the center of atom is, a mathematical method involving Gaussian Fitting based on Matlab is essential to ascertain the accurate position of every atom. Gaussian Fitting could give an accurate position of atom according to the brightness of every atom.

Determining atomic positions by fitting each atom site using a spherical Gaussian algorithm in Matlab is a common method. For double-check of our conclusions, we used two different softwares: CalAtom ${ }^{36,37}$ and StatSTEM ${ }^{38}$ and compare their calculating results. The average value distribution of data using these two softwares are keeping an exact consistency. For example, for the Fe-Fe bond length of strong ladder at room temperature, CalAtom reveals that they are $2.71 \AA$ and $2.55 \AA$; the StatSTEM reveals that they are $2.72 \AA$ and $2.54 \AA$ Besides, each bond length acquired from atoms-position-determination software are based on the statistics value of about 300 data, thus they are statistically meaningful. Furthermore, multiple images were recorded for most of the data we exhibit. The multiple images were averaged in order to reduce noise and artifacts induced by possible random drifts of the sample. In the average procedure, the aliment of these images was done by minimizing the shift of an individual image relative to the averaged image using an iterative rigid alignment method. Another methods to measure the artifacts in STEM images is to determine the displacements of atoms in which no offcenter displacements would happen ${ }^{39}$. On the basis of this method, Lu, L. et al. measured the STO layers ${ }^{39}$ with $\mathrm{rms}=4.8 \mathrm{pm}$, we observed the STO layers with $\mathrm{rms}=3.2 \mathrm{pm}$ using CalAtom software.

\section{DATA AVAILABILITY}

The datasets generated during this study are available from the corresponding author on reasonable request.

Received: 8 May 2020; Accepted: 28 June 2020; Published online: 23 July 2020

\section{REFERENCES}

1. Pulvari, C. F. Ferrielectricity. Phys. Rev. 120, 1670-1673 (1960).

2. Miller, R. C., Wood, E. A., Remeika, J. P. \& Savage, A. Na( $\left(\mathrm{Nb}_{1-\mathrm{x}} \mathrm{V}_{\mathrm{x}}\right) \mathrm{O}_{3}$ system and "ferrielectricity". J. Appl. Phys. 33, 1623-1630 (1962).

3. Scott, J. F. et al. Ferrielectricity in the metal-organic ferroelectric tris-sarcosine calcium chloride. Phys. Rev. B 95, 094119 (2017).

4. Maisonneuve, V., Cajipe, V. B., Simon, A., Muhll, R. V. D. \& Ravez, J. Ferrielectric ordering in lamellar CulnP ${ }_{2} S_{6}$. Phys. Rev. B 56, 10860-10868 (1997).

5. Liu, F. et al. Room-temperature ferroelectricity in Culn $\mathrm{P}_{2} \mathrm{~S}_{6}$ ultrathin flakes. Nat. Commun. 7, 12357 (2016).

6. Gou, G. \& Rondinelli, J. M. Piezoelectricity across a strain-induced isosymmetric ferri-to-ferroelectric transition. Adv. Mater. Inter. 1, 311-316 (2015).

7. Pitcher, M. J. et al. Tilt engineering of spontaneous polarization and magnetization above $300 \mathrm{~K}$ in a bulk layered perovskite. Science 347, 420-424 (2015).

8. Spaldin, N. A., Fiebig, M. \& Mostovoy, M. The toroidal moment in condensedmatter physics and its relation to the magnetoelectric effect. J. Phys. Conden. Matter 20, 2709-2713 (2008). 
9. Oh, Y. S., Luo, X., Huang, F. T., Wang, Y. \& Cheong, S. W. Experimental demonstration of hybrid improper ferroelectricity and the presence of abundant charged walls in $\left(\mathrm{Ca}, \mathrm{Sr}_{3} \mathrm{Ti}_{2} \mathrm{O}_{7}\right.$ crystals. Nat. Mater. 14, 407-413 (2015).

10. Orlandi, F. et al. Structural and electric evidence of ferrielectric state in $\mathrm{Pb}_{2} \mathrm{MnWO}_{6}$ double perovskite system. Inorg. Chem. 453, 10283-10290 (2015).

11. Dai, P., Hu, J. \& Dagotto, E. Magnetism and its microscopic origin in iron-based high-temperature superconductors. Nat. Phys. 8, 709-718 (2012).

12. Ying, J., Lei, H., Petrovic, C., Xiao, Y. \& Struzhkin, V. V. Interplay of magnetism and superconductivity in the compressed Fe-ladder compound $\mathrm{BaFe}_{2} \mathrm{Se}_{3}$. Phys. Rev. $B$ 95, 241109 (R) (2017).

13. Zhang, Y., Lin, L. F., Zhang, J. J., Dagotto, E. \& Dong, S. Sequential structural and antiferromagnetic transitions in $\mathrm{BaFe}_{2} \mathrm{Se}_{3}$ under pressure. Phys. Rev. B 97, 045119 (2018).

14. Dong, S., Liu, J.-M. \& Dagotto, E. BaFe $\mathrm{Se}_{3}$ : A high TC magnetic multiferroic with large ferrielectric polarization. Phys. Rev. Lett. 113, 187204 (2014)

15. Caron, J. M., Neilson, J. R., Miller, D. C., Llobet, A. \& McQueen, T. M. Iron displacements and magnetoelastic coupling in the antiferromagnetic spin-ladder compound $\mathrm{BaFe}_{2} \mathrm{Se}_{3}$. Phys. Rev. B 84, 180409 (R) (2011).

16. Svitlyk, V. et al. Crystal structure of $\mathrm{BaFe}_{2} \mathrm{Se}_{3}$ as a function of temperature and pressure: phase transition phenomena and high-order expansion of Landau potential. J. Phys. Conden. Matter 25, 315403 (2013).

17. Nambu, Y. et al. Block magnetism coupled with local distortion in the iron-based spin-ladder compound $\mathrm{BaFe}_{2} \mathrm{Se}_{3}$. Phys. Rev. B 85, 064413 (2012).

18. Lei, H., Ryu, H., Frenkel, A. I. \& Petrovic, C. Anisotropy in $\mathrm{BaFe}_{2} \mathrm{Se}_{3}$ single crystals with double chains of FeSe tetrahedra. Phys. Rev. B 84, 214511 (2011).

19. Krztonmaziopa, A. et al. The synthesis, and crystal and magnetic structure of the iron selenide $\mathrm{BaFe}_{2} \mathrm{Se}_{3}$ with possible superconductivity at $\mathrm{Tc}=11 \mathrm{KJ}$. Phys. Condens. Matter 23, 402201 (2011).

20. Tian, $\mathrm{H}$. et al. Interface-induced modulation of charge and polarization in thin film $\mathrm{Fe}_{3} \mathrm{O}_{4}$. Adv. Mater. 26, 461-465 (2014).

21. Coey, M. Condensed-matter physics: charge-ordering in oxides. Nature 430 155-157 (2004).

22. Lin, L.-F. et al. Ferroelectric ferrimagnetic $\mathrm{LiFe}_{2} \mathrm{~F}_{6}$ : charge ordering mediated magnetoelectricity. Phys. Rev. Mater. 1, 071401(R) (2017).

23. Caron, J. M. et al. Orbital-selective magnetism in the spin-ladder iron selenides $\mathrm{Ba}_{1-\mathrm{x}} \mathrm{K}_{\mathrm{x}} \mathrm{Fe}_{2} \mathrm{Se}_{3}$. Phys. Rev. B 85, 180405 (R) (2012).

24. $\mathrm{Li}, \mathrm{W}$. et al. Phase separation and magnetic order in $\mathrm{K}$-doped iron selenide superconductor. Nat. Phys. 8, 126-130 (2012).

25. Li, W., Dong, S., Fang, C. \& Hu, J.-P. Block antiferromagnetism and checkerboard charge ordering in the alkali-doped iron selenides $\mathrm{R}_{1-\mathrm{x}} \mathrm{Fe}_{2-\mathrm{y}} \mathrm{Se}_{2}$. Phys. Rev. $B$ 85, 100407 (R) (2012).

26. King-Smith, R. D. \& Vanderbilt, D. Theory of polarization of crystalline solids. Phys. Rev. B 47, 1651-1654 (1993).

27. Aoyama, T. et al. Polar state induced by block-type lattice distortions in $\mathrm{BaFe}_{2} \mathrm{Se}_{3}$ with quasi-one-dimensional ladder structure. Phys. Rev. B 99, 241109 (R) (2019).

28. Zheng, W. et al. Room temperature polar structure and multiferroicity in BaF$\mathrm{e}_{2} \mathrm{Se}_{3}$. Phys. Rev. B 101, 020101 (R) (2020).

29. Hur, N. et al. Electric polarization reversal and memory in a multiferroic material induced by magnetic fields. Nature 429, 392-395 (2004).

30. Leo, N. et al. Independent ferroelectric contributions and rare-earth-induced polarization reversal in multiferroic $\mathrm{TbMn}_{2} \mathrm{O}_{5}$. Phys. Rev. B 85, 094408 (2012).

31. Lin, L.-F., Zhang, Y., Meoro, A., Dagotto, E. \& Dong, S. Frustrated dipole order induces noncollinear proper ferrielectricity in two dimensions. Phys. Rev. Lett. 123, 067601 (2019).

32. Kresse, G. \& Hafner, J. Ab initio molecular dynamics for liquid metals. Phys. Rev. $B$ 47, 558 (1993).

33. Kresse, G. \& Furthmüller, J. Efficient iterative schemes for ab initio total-energy calculations using a plane-wave basis set. Phys. Rev. B 54, 11169-11186 (1996).

34. Perdew, J. P., Burke, K. \& Ernzerhof, M. Generalized gradient approximation made Simple. Phys. Rev. Lett. 77, 3865 (1996).

35. King-Smith, R. D. \& Vanderbilt, D. Theory of polarization of crystalline solids. Phys. Rev. B 47, 1651-1654 (1993).

36. Zhang, Q. et al. Multiple-ellipse fitting method to precisely measure the positions of atomic columns in a transmission electron microscope image. Micron 113, 99-104 (2018).

37. Zhang, Q. et al. CalAtom: A software for quantitatively analysing atomic columns in a transmission electron microscope image. Ultramicroscopy 202, 114-120 (2019).
38. De Backer, A., Van den Bos, K. H. W., Van den Bos, W., Sijbers, J. \& Van Aert, S StatSTEM: an efficient approach for accurate and precise model-based quantification of atomic resolution electron microscopy images. Ultramicroscopy $\mathbf{1 7 1}$ 104-116 (2016)

39. Lu, L. et al. Topological defects with distinct dipole configurations in $\mathrm{PbTiO} /$ SrTiO3 multilayer films. Phys. Rev. Lett. 120, 177601 (2018).

\section{ACKNOWLEDGEMENTS}

We acknowledge the National Natural Science Foundation of China (Grant Nos 11834002, 11674055, and 11234011), National Key R\&D Program of China 2017YFB0703100, and the 111 Project (Grant No. B16042). K.D. acknowledges the China Scholarship Council (CSC, No.201806320230) for sponsorship and 2019 Zhejiang University Academic Award for Outstanding Doctoral Candidates. We thank Prof. Fang Lin for providing guidance on calculating atoms position and Dr. Andrew Studer for performing neutron powder diffraction. We thank Prof. SangWook Cheong, Prof. Zhigao Sheng, Prof. Qianghua Wang, Prof. Meng Wang, Prof Renkui Zheng, Prof. Takuya Aoyama, Dr. Zhibo Yan, and Dr. Meifeng Liu for valuable discussion and/or technical help during measurements.

\section{AUTHOR CONTRIBUTIONS}

H.T., S.D., Z.Z., and G.V.T. co-designed the project. K.D. performed the experiments related to electron microscopy. K.D. and X.C. conduct the in-situ heating experiments. K.D. and Z.N.Z. designed the displacement vector-mapping algorithm for the statistics of bond length. L.G. and Y.L. synthesized the samples. L.G., J.P., Y.L., T.Z., J.L., and Z.N. measured macroscopic physical properties. Y.Z. and S.D. did DFT calculations. S.D. figured out the Laudau theory formula. S.D., J.P., and S.S.W analyzed the symmetry. K.D., S.D., H.T., and G.V.T. co-write the paper. All authors contributed to the discussions and manuscript preparation. K.D., L.G., and J.P. contributed equally.

\section{COMPETING INTERESTS}

The authors declare no competing interests.

\section{ADDITIONAL INFORMATION}

Supplementary information is available for this paper at https://doi.org/10.1038/ s41535-020-00252-y.

Correspondence and requests for materials should be addressed to S.D. or H.T.

Reprints and permission information is available at http://www.nature.com/ reprints

Publisher's note Springer Nature remains neutral with regard to jurisdictional claims in published maps and institutional affiliations.

Open Access This article is licensed under a Creative Commons Attribution 4.0 International License, which permits use, sharing, adaptation, distribution and reproduction in any medium or format, as long as you give appropriate credit to the original author(s) and the source, provide a link to the Creative Commons license, and indicate if changes were made. The images or other third party material in this article are included in the article's Creative Commons license, unless indicated otherwise in a credit line to the material. If material is not included in the article's Creative Commons license and your intended use is not permitted by statutory regulation or exceeds the permitted use, you will need to obtain permission directly from the copyright holder. To view a copy of this license, visit http://creativecommons. org/licenses/by/4.0/.

(c) The Author(s) 2020 